\title{
miR-222 promotes invasion and migration of ovarian carcinoma by targeting PTEN
}

\author{
LI GONG $^{1}$, WEIMIN ZHANG ${ }^{2}$, YUCHAO YUAN ${ }^{3}, \mathrm{XIA} \mathrm{XING}^{3}, \mathrm{HUI} \mathrm{LI}^{4}$ and GUIFENG ZHAO ${ }^{5}$ \\ ${ }^{1}$ Department of Gynaecology, People's Hospital of Rizhao, Rizhao, Shandong 276826; ${ }^{2}$ Department of Obstetrics and \\ Gynecology, Zhangqiu Maternal and Child Health Care Hospital, Jinan, Shandong 250000; Departments of \\ ${ }^{3}$ Obstetrics and Gynecology, and ${ }^{4}$ Rehabiliation Medicine, People's Hospital of Zhangqiu, Jinan, Shandong 250200; \\ ${ }^{5}$ Department of Surgery, People's Hospital of Weifang, Weifang, Shandong 261000, P.R. China
}

Received November 30, 2017; Accepted April 30, 2018

DOI: $10.3892 / \mathrm{ol} .2018 .8743$

\begin{abstract}
Ovarian carcinoma is the most lethal of the gynecologic malignancies worldwide. Increasing evidence suggests dysfunction of microRNAs (miRNAs) plays an important role in human cancers. The function of miR-222 was detected in ovarian carcinoma to verify the regulation of phosphatase and tensin homolog (PTEN) by miR-222. miR-222 expression in ovarian carcinoma tissues and cell lines were examined using RT-qPCR. Transwell assay was used to detect miR-222 effects on ovarian carcinoma cell migration and invasion. Western blot analysis and luciferase assays were performed to validate PTEN as miR-222 targets. miR-222 expression was upregulated in ovarian carcinoma tissues and three cell lines (A2780, SKOV-3 and OVCAR-3). Ectopic overexpression of miR-222 in ovarian carcinoma cells was sufficient to promote invasion and migration. PTEN acted as a direct target of miR-222. Overexpression of PTEN inhibited human ovarian carcinoma cell migration and invasion. In summary, our findings suggest that miR-222 plays an important role in promoting ovarian carcinoma cell invasion and migration and miR-222/PTEN may be a novel therapeutic target of miRNA-mediated promotion of cell invasion and migration in ovarian carcinoma.
\end{abstract}

\section{Introduction}

Ovarian carcinoma is the most lethal of the gynecologic malignancies in women worldwide (1). Epithelial ovarian carcinoma (EOC), which accounts for $90 \%$ (2) of ovarian carcinoma and for $4.2 \%$ of all cancer deaths in women globally, is a heterogeneous group of neoplasms $(3,4)$. Due to several factors of insidious nature, manifesting with little or no symptoms until the disease progresses to metastasis, along with a wide diversity

Correspondence to: Dr Guifeng Zhao, Department of Surgery, People's Hospital of Weifang, 151 Guangwen Street, Weifang, Shandong 261000, P.R. China

E-mail: zsej69052@126.com

Key words: miR-222, ETS1, ovarian carcinoma of histological subtypes and corresponding clinical behavior, poses significant therapeutic challenges (5). Although significant progress in the diagnosis and treatment including surgery, radiotherapy and chemotherapy have been made in the treatment of ovarian carcinoma, the 5-year survival rate for all stages is below $45 \%$ and is decreased to $25 \%$ among patients with advanced ovarian carcinoma $(6,7)$. Inability to prolong patient remission is a critical gap in the clinical management of ovarian carcinoma (5). The underlying cause of this problem stems in part from insufficient basic knowledge of the biology and mechanisms supporting ovarian carcinoma carcinogenesis and progression. Therefore, it is crucial to find the underlying molecular mechanisms of ovarian carcinoma and identify the promising therapeutic targets to improve treatment strategies.

MicroRNAs (miRNAs) are a class of small, highly conserved, non-coding RNAs with a length of approximately 21-24 nucleotides that bind to the 3'-untranslated region (3'-UTR) of target messenger RNAs (mRNAs) to induce translational degradation or repression (8). Given the ability of miRNAs to regulate gene expression (9), they unsurprisingly became the critical point in their involvement in cancer. Increasing evidence indicated that miRNAs are frequently dysregulated in cancers (10-12) where they have been shown to contribute to disease pathogenesis, as well as cancer cell death, differentiation, proliferation, metastasis, and apoptosis (13-15). Previous investigations have identified the dysregulated expression of various miRNAs involved in human ovarian carcinoma tumorigenesis and progression, such as cell proliferation, metastasis, migration and DNA methylation $(16,17)$. Among such a large number of miRNAs that have been identified as having significant roles in tumorigenesis, miR-222 was identified as a key miRNA, located on human chromosome Xp11.3 as a single transcript, and showing high sequence identity (18). Functional studies showed that miR-222 reinforced cancer cell biological progression (19), promoted tumor cell proliferation, migration and microtubule formation, and the novel regulatory axis miR-222-3p/GNAI2/ AKT is a potential therapeutic target for EOC patients in the future (20). Although the importance of miRNAs in cancer pathological processes have attracted much attention in recent decades, the pathological relevance and significance of the majority miRNAs in ovarian carcinoma remains unclear. 
Table I. Clinicopathological variables and miR-200c expression in 40 ovarian carcinoma patients.

\begin{tabular}{|c|c|c|c|c|}
\hline \multirow[b]{2}{*}{ Characteristics } & \multirow[b]{2}{*}{ Total cases $(n=40)$} & \multicolumn{2}{|c|}{ miR-200c expression } & \multirow[b]{2}{*}{ P-value } \\
\hline & & Low $(\%)$ & High $(\%)$ & \\
\hline Age (years) & & & & $P>0.05$ \\
\hline$\leq 40$ & 18 & $8(71.43)$ & $10(28.57)$ & \\
\hline$>40$ & 22 & $10(69.23)$ & $12(30.77)$ & \\
\hline Tumor size (cm) & & & & $P>0.05$ \\
\hline$<5$ & 18 & $12(66.67)$ & $6(33.33)$ & \\
\hline$\geq 5$ & 22 & $6(72.73)$ & $16(27.27)$ & \\
\hline Histological grading & & & & $\mathrm{P}<0.01$ \\
\hline $1-2$ & 31 & $13(90.32)$ & $18(9.68)$ & \\
\hline 3 & 9 & $5(55.56)$ & $4(44.44)$ & \\
\hline Distant metastasis & & & & $\mathrm{P}<0.05$ \\
\hline Yes & 25 & $9(90.00)$ & $16(10.00)$ & \\
\hline No & 15 & $9(63.33)$ & $6(36.67)$ & \\
\hline FIGO stage & & & & $\mathrm{P}<0.01$ \\
\hline I-II & 29 & $12(41.38)$ & $17(58.62)$ & \\
\hline III-IV & 11 & $6(76.47)$ & $5(23.53)$ & \\
\hline
\end{tabular}

Phosphatase and tensin homolog $(P T E N)$ is well recognized as tumor suppressor gene and is involved in the regulation of cancer cell biological behavior. PTEN could antagonize PI3 kinase (PI3K) activity by converting phosphatidylinositol $(3,4,5)$-trisphosphate in the cytoplasm into phosphatidylinositol (4,5)-bisphosphate. The PI3K dysregulation can result in Akt hyper-activation, and promote tumor cell radioresistance, proliferation, migration, and invasion. PTEN has been identified as one direct target of miR-222 (21), we thus reasoned miR-222 may promote the invasion and migration by targeting PTEN of ovarian carcinoma, and be responsible for ovarian carcinoma progression and tumorigenesis.

In order to explore the regulatory mechanism whereby miR-222 was involved in ovarian carcinoma cell migration and invasion, we proved miR-222 could positively regulate PTEN expression and confirmed miR-222 functioned as a vital part in cell migration and invasion of ovarian carcinoma regulated by PTEN. These results may indicate a novel regulatory mechanism for cell migration and invasion in ovarian carcinoma and provide a new avenue for exploring ovarian carcinogenesis.

\section{Materials and methods}

Clinical specimens. Forty paired tissues and the adjacent normal tissues (ANT) of ovarian carcinoma were collected at the People's Hospital of Rizhao (Rizhao, China), from 2013 to 2015. Patients did not receive radiotherapy or chemotherapy priro to surgery. The Ethics Committee of People's Hospital of Rizhao approved the present study. All samples were obtained with written informed consent from participating patients. All the tissues obtained from biopsy or surgery were frozen at $-80^{\circ} \mathrm{C}$. The clinical characteristics are shown in Table I.

Cell culture. Ovarian carcinoma cell lines A2780, SKOV-3 and OVCAR-3 and human ovarian surface epithelial cell line
(HOSEpiC) were obtained from the Shanghai Institute of Cell Biology (Shanghai, China). Ovarian carcinoma cell lines were cultured in Dulbecco's modified Eagle's medium (DMEM; HyClone; GE Healthcare, Chicago, IL, USA). After the cells adhered, transfection of the miR-222 mimics/inhibitor/negative control (NC) were performed with Lipofectamine ${ }^{\mathrm{TM}} 2000$ (Invitrogen; Thermo Fisher Scientific, Inc., Waltham, MA, USA), which achieved the ectopic expression of miRNA. The transfection protocol for siRNA was the same as that for miR-222 mimics/inhibitor. The miR-222 mimics/ inhibitor, pGL3-PTEN 3'UTR and pGL3-NC were obtained from Guangzhou RiboBio Co., Ltd. (Guangzhou, China). The sequences of miR-222 mimics/inhibitor were: miR-222 mimic, AGCUACAUCUGGCUACUGGGU; and inhibitor, GCGAUGUAGACCGAUGACCA.

Reverse transcription-quantitative PCR (RT-qPCR). Total RNA was extracted from culture cells and cancer tissues using the TRIzol reagents (Ambion; Thermo Fisher Scientific, Inc.). PTEN was relative to $\beta$-actin and miR-222 was normalized relative to U6 endogenous control using the $2^{-\Delta \Delta C q}$ method. The primers used were: miR-222 forward, 5'-AGC TACATCTGGCTACTGG-3', and reverse, 5'-GTATCCAGT GCAGGGTCC-3'; U6 forward, 5'-CTCGCTTCGGCAGC ACA-3' and reverse, 5'-TGGTGTCGTGGAGTCG-3'. PTEN forward 5'-TGGCGGAACTTGCAATCCTCAGT-3' and reverse, 5'-TCCCGTCGTGTGGGTCCTGA-3'. $\beta$-actin forward, 5'-TTGCCGACAGGATGCAGAAGGA-3' and reverse, 5'-AGGTGGACAGCGAGGCCAGGAT-3'.

Transwell assay. Cell Transwell assays were conducted using chambers (Costar, NY) with or without $2 \mathrm{mg} / \mathrm{ml}$ Matrigel (Clontech Laboratories, Inc., Mountainview, CA, USA). OVCAR-3 and SKOV-3 cells were transfected with the miR-222 mimic /inhibitor and NC and isolated to make a final concentration at $2 \times 10^{5} / \mathrm{ml}$, then placed into the upper chamber. 
A

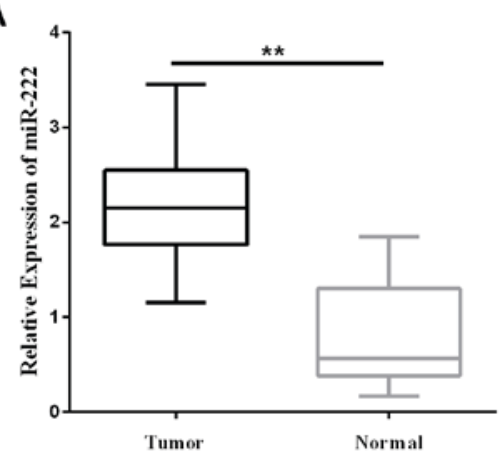

C

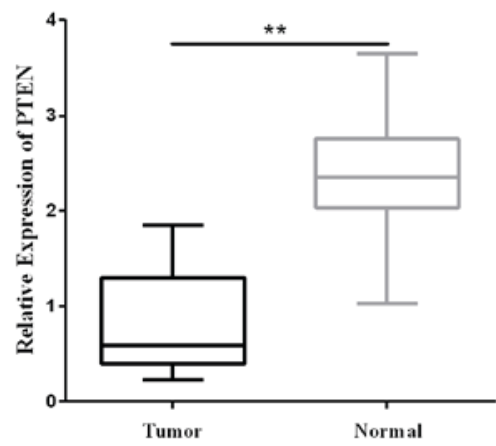

B

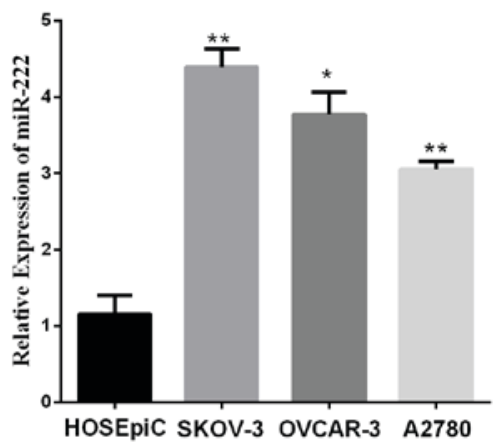

D

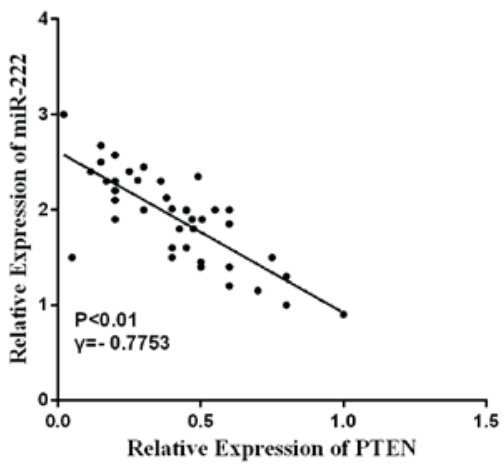

Figure 1. miR-222 expression is upregulated in ovarian carcinoma tissues and cell lines and inversely correlated with PTEN. (A) miR-222 expression in 40 pairs of ovarian carcinoma samples and their ANT. The expression level of miR-222 was detected by RT-qPCR using U6 as the internal control. (B) miR-222 expression levels in SKOV-3, A2780 and OVCAR-3 cancer cell lines and primary normal ovarian carcinoma squamous cells (HOSEpiC). (C) Relative mRNA expression of PTEN in ovarian carcinoma tissues. (D) Spearman's correlation analysis of miR-222 expression and PTEN in 40 ovarian carcinoma tissues $(\gamma=-0.7753, \mathrm{P}<0.01) .{ }^{* *} \mathrm{P}<0.01,{ }^{*} \mathrm{P}<0.05$. PTEN, phosphatase and tensin homolog; ANT, adjacent normal tissues; RT-qPCR, reverse-transcription quantitative PCR; HOSEpiC, human ovary surface epithelial cell line.

Lower chambers were filled with medium containing $20 \%$ fetal bovine serum as a chemo-attractant. The cells were fixed for $15 \mathrm{~min}$ using $1 \mathrm{ml} /$ well $4 \%$ paraformaldehyde, stained with Giemsa (JRDUN Biotechnology Co. Ltd., Shanghai, China) for $30 \mathrm{~min}$, and washed three times with $1 \mathrm{X}$ phosphate-buffered saline. Finally, stained cells were counted under a microscope (Olympus, Tokyo, Japan).

Western blot analysis. Cultured cells were collected and lysed with radioimmune precipitation assay lysis buffer (Beyotime Institute of Biotechnology, Haimen, China). Protein samples were transferred to polyvinyl difluoride membranes (EMD Millipore, Billerica,MA,USA), and the membranes were incubated in primary antibodies rabbit monoclonal anti-PTEN (ab109454; 1:1,000; Abcam, Cambridge, MA, USA) followed by incubation with horseradish peroxidase-coupled secondary antibody goat anti-rabbit IgG-HRP (sc-2004; 1:3,000; Santa Cruz Biotechnology, Inc., Santa Cruz, CA, USA). $\beta$-actin was used as internal control. ImageJ software (NIH, Bethesda, MD, USA) was used to quantify the protein bands.

Luciferase reporter. The PTEN mutant (MT) and wild-type (WT) 3'UTR were cloned from human genomic DNA. Cells were seeded on 24-well plates for luciferase assay. miR-222 mimic plus PTEN WT or MT 3'UTR were transfected into OVCAR-3 and SKOV-3. Then, we used Luciferase Reporter
Assay (Promega Corporation, Madison, WI, USA) according to the manufacturer's protocols.

Statistical analysis. Statistical results were analyzed by SPSS 16.0 software (SPSS, Inc., Chicago, IL, USA) statistics package. The results are presented as the mean \pm standard deviation (SD). The Student's t-test was used to analyze experimental data. The unpaired two group comparison and multiple comparisons were made with analysis of variance (ANOVA) with Tukey-Kramer post hoc test. Correlation between mRNA and miRNA was estimated using the Spearman's correlation method. $\mathrm{P}<0.05$ was considered to indicate a statistically significant difference.

\section{Results}

miR-222 expression is upregulated and inversely correlates with PTEN in ovarian carcinoma. The miR-222 expression levels were detected in 40 matched pairs of ovarian carcinoma tissues and ANT by RT-qPCR. In comparison to the normal cervical tissues, miR-222 expression was significantly higher in the ovarian carcinoma tissues (Fig. 1A; $\mathrm{P}<0.01$ ). To investigate potential associations between miR-222 expression and patient's clinicopathological variables, we divided the patients with ovarian carcinoma into two groups based on mean value (2.200) of miR-222 expression: High expression 
A

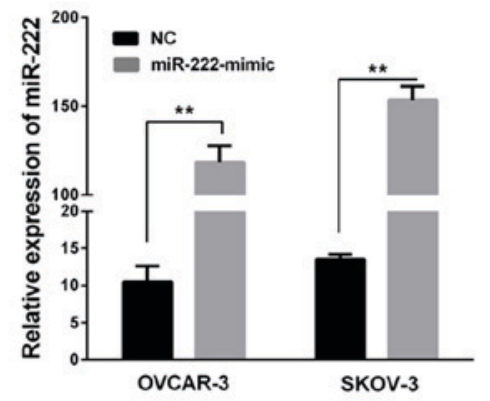

C
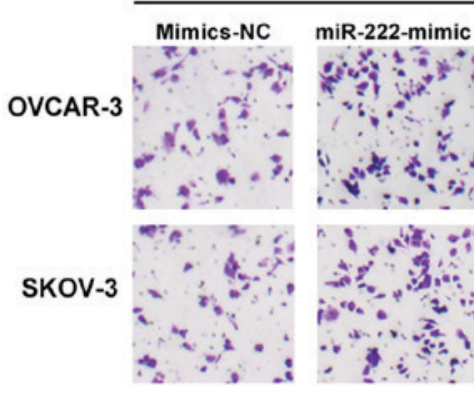

Migration

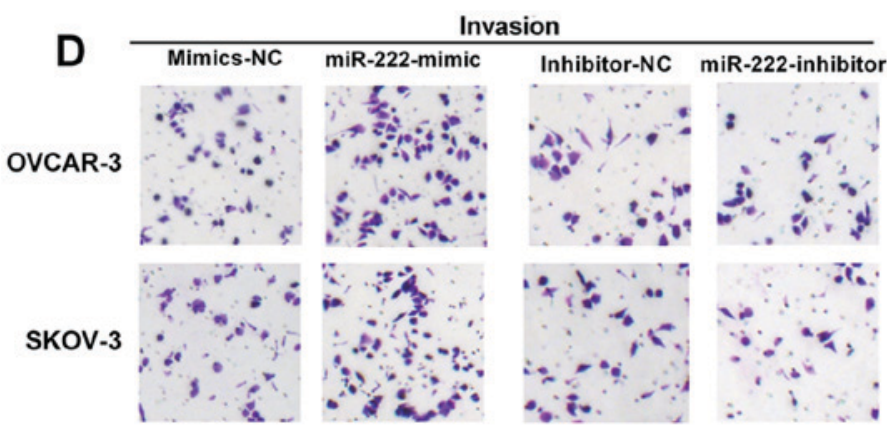

B
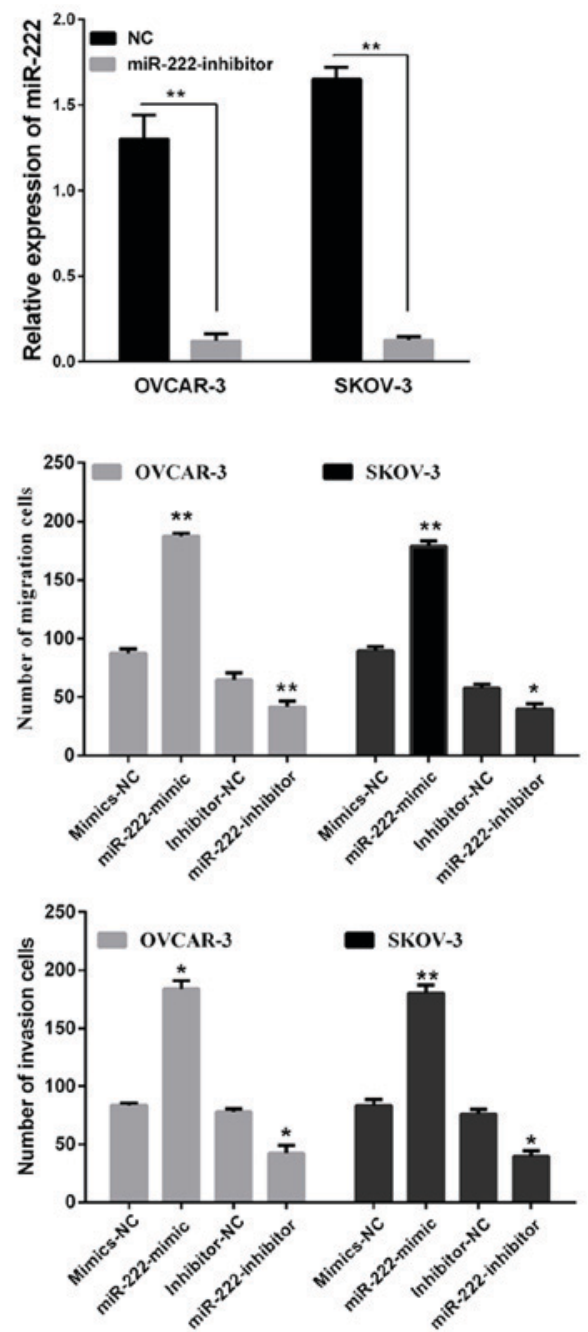

Figure 2. miR-222 promotes invasion and migration of ovarian carcinoma in vitro. (A and B) miR-222 was normally expressed in OVCAR-3 and SKOV-3 and miR-222 levels were determined by RT-qPCR after transfected miR-222 mimic/inhibitor and negative control (NC). (C and D) Transwell assay showed that miR-222 promotes invasion and migration of OVCAR-3 and SKOV-3 cells after transfection of miR-222 mimic/inhibitor or miR-ctrl. ${ }^{* *} \mathrm{P}<0.01$, $\mathrm{P}<0.05$. RT-qPCR, reverse transcription-quantitative PCR; NC, negative control.

$(>2,200, n=18)$, and low expression $(<2,200, n=22)$ groups Statistical analysis suggested that a high miR-222 expression level correlated with histological grading, distant metastasis, and FIGO stage, whereas no statistical difference was found in the correlation of miR-222 expression with age and tumor size (Table I). In addition to ovarian carcinoma tissue samples, we selected three cell lines (A2780, SKOV-3 and OVCAR-3) compared with the normal cells (HOSEpiC) to further detect miR-222 relative expression levels. The results showed the miR-222 levels in A2780, SKOV-3 and OVCAR-3 were significantly increased relative to that in the normal cell HOSEpiC (Fig. 1B, P<0.05).

Furthermore, the levels of PTEN in the ovarian carcinoma tissues and the ANT were assessed using RT-qPCR. The PTEN relative expression level was significantly decreased in the ovarian carcinoma tissues, as shown in Fig. $1 \mathrm{C}(\mathrm{P}<0.01)$. miR-222 was negatively correlated with the PTEN level in these clinical specimens (Fig. 1D). These results indicated miR-222 is upregulated in ovarian carcinoma tissues and that the increase of expression of miR-222 decreased PTEN level, which may play an essential part in the progression of ovarian carcinomas. Additionally, miR-222 may be critical in regulating ovarian carcinoma cell invasion and migration. However, the particular function of miR-222 ovarian carcinoma is unknown.

miR-222 promotes migration and invasion of ovarian carcinoma. To reveal the functional role of miR-222 in affecting the migration and invasion of ovarian carcinoma, SKOV-3 and OVCAR-3 cell lines were both transfected with the miR-222 mimic/inhibitor/NC. The results showed SKOV-3 and OVCAR-3 were transfected with miR-222 mimics both expressed at a relatively high level compared with cell lines transfected corresponding to negative control (Fig. 2A). However, miR-222 expression level of cell lines transfected with miR-222 inhibitor were relatively lower than cells transfected with corresponding negative control (Fig. 2B). Transwell assays without Matrigel were used to examine the miR-222 function on the cell migratory potential, and Transwell assays with Matrigel were used to detect the miR-222 effects on the cell invasive potential. Overexpression of miR-222 significantly increased the migration and invasion capacities in the OVCAR-3 and SKOV-3 cells (Fig. 2C). Similarly, Fig. 2D showed re-expression of miR-222 in OVCAR-3 and SKOV-3 
A

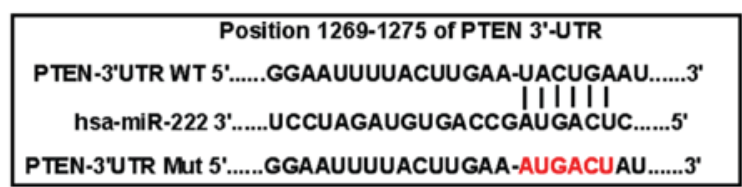

C

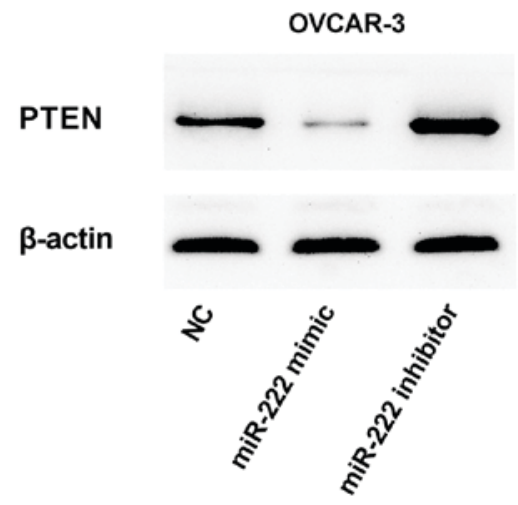

B

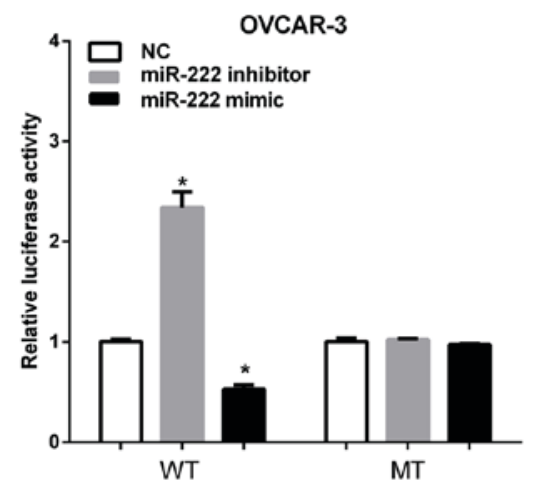

D

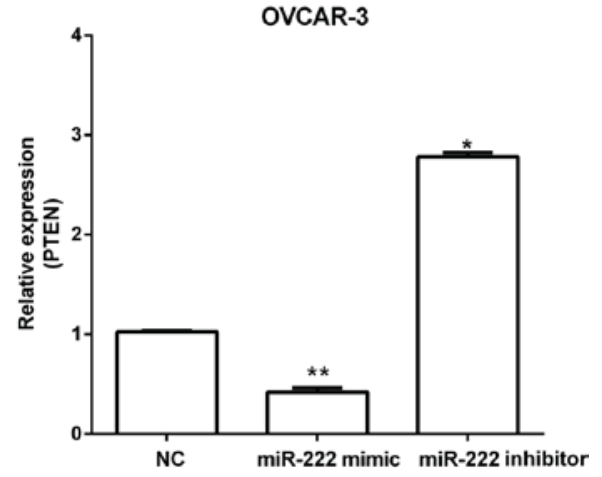

Figure 3. miR-222 suppresses PTEN expression by targeting PTEN 3'-UTR. (A) The binding sites of miR-222 on PTEN 3'-UTR is predicted by TargetScan. (B) Luciferase reporter assay of OVCAR-3 with the pGL- PTEN-3'-UTR-WT or pGL- PTEN-3'-UTR-MT were co-transfected with miR-506 mimics/inhibitor. (C and D) PTEN protein expression in OVCAR-3 transfected with miR-222 mimics/inhibitor or negative control was detected by western blot analysis. "P<0.05; ${ }^{* *} \mathrm{P}<0.01$. PTEN, phosphatase and tensin homolog.

A

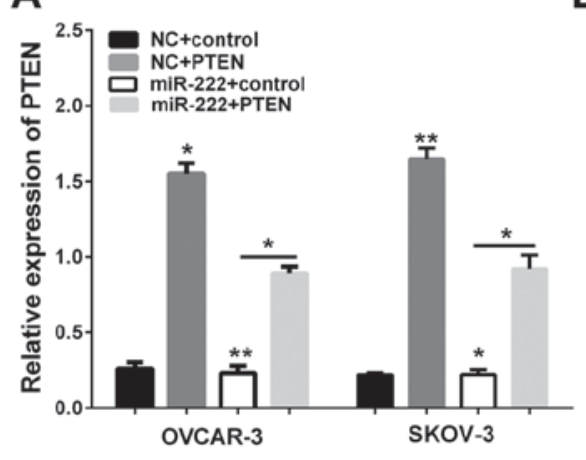

B

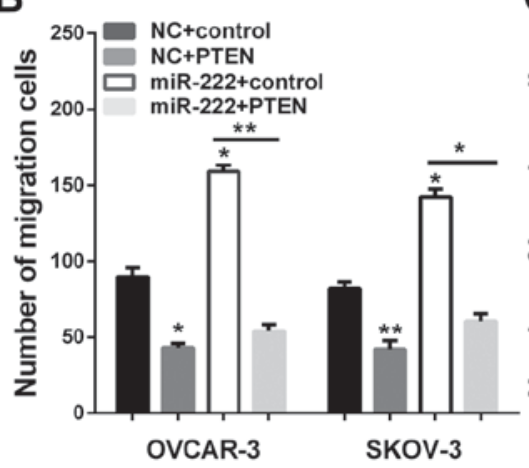

C

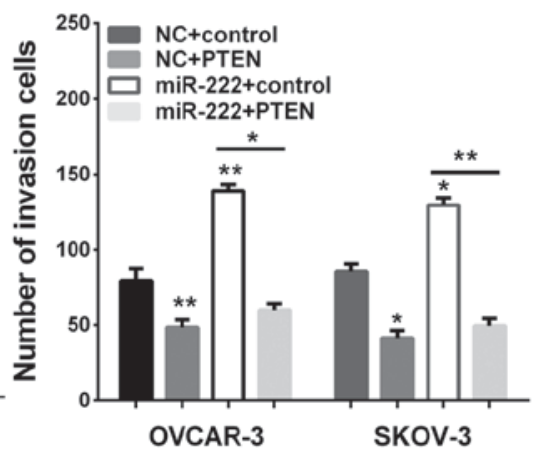

Figure 4. Overexpression of PTEN inhibits migration and invasion of ovarian carcinoma. (A) PTEN was abnormally expressed in OVCAR-3 and SKOV-3 and PTEN levels were determined by RT-qPCR. (B) Overexpression of PTEN significantly inhibited the migration ability of OVCAR-3 and SKOV-3 cells. (C) Overexpression of PTEN significantly inhibited the invasiveness of OVCAR-3 and SKOV-3 cells. * $\mathrm{P}<0.05 ;{ }^{* *} \mathrm{P}<0.01$. PTEN, phosphatase and tensin homolog; RT-qPCR, reverse transcription-quantitative PCR.

cells transfected with the miR-222 mimic/inhibitor compared with corresponding NC. In summary, these findings indicated that miR-222 may act as an oncomiR and promotes cell migration and invasion during ovarian carcinoma progression.

PTEN is a target and downregulated by miR-222. Predictive tools (TargetScan Huma http://www.targetscan.org/vert_71/) were used to find target genes of miR-222. The miR-222 putative binding sites were found in the 3'-UTR of PTEN at 1269-1275 bp (Fig. 3A). To determine whether miR-222 can regulate PTEN expression, the WT plasmids pGL-UTR and MT plasmid pGL-mUTR of PTEN 3'-UTR were constructed based on luciferase reporter assay, which were then both transfected into OVCAR-3 with or without miR-222 overexpression. Decreased expression of PTEN was observed after transfecting by miR-222 mimic. However, miR-222 inhibitor increased PTEN expression (Fig. 3B). Furthermore, the PTEN mRNA and protein expression were increased by miR-222 inhibitor, and miR-222 mimic had the opposite effect on PTEN expression (Fig. 3C and D). 
Overexpression of PTEN promotes migration and invasion of ovarian carcinoma. Having confirmed that miR-222 acts to reduce PTEN levels in ovarian carcinoma, we then determined whether PTEN is involved in the migration and invasion of ovarian carcinoma. We therefore transfected the control group and PTEN plasmid into the miR-222 cells and NC for Transwell assay. The successful overexpression of PTEN was verified by RT-qPCR (Fig. 4A). We further analyzed the migration and invasion of OVCAR-3 and SKOV-3. We found that an increase of the expression of PTEN could effectively reverse the promotion effect on cell migration and invasion induced by miR-222 overexpression in OVCAR-3 and SKOV-3 (Fig. 4B and C). These findings suggest that PTEN is responsible for the tumorigenic effects in ovarian carcinoma cells, and PTEN may be important in migration and invasion of ovarian carcinoma cells.

\section{Discussion}

A number of miRNAs have been proved to be encoded in ovarian carcinoma progression and development through effect on cancer cell proliferation, migration, apoptosis, and invasion $(16,17)$. For example, microRNA-223-3p promoted cell growth and invasion through targeting SOX11 expression of ovarian carcinoma (22). Xu et al demonstrated that miR-28-5p promotes the progression and development of ovarian carcinoma through inhibition of N4BP1 (23). Thus, identification of cancer-specific miRNAs and their involved targets is pivotal for comprehending their function in tumor migration and invasion, and to provide critical clues for diagnosis and therapy of ovarian carcinoma.

Previous studies have suggested miR-222 was altered in ovarian carcinoma and its functional role was extremely tangled as it could act as promising target for therapeutic purposes (20). miR-222 was significantly increased in ovarian carcinoma cell lines and tissues, and its expression was positively correlated with PTEN. Further studies revealed that miR-222 promoted ovarian carcinoma cell migration and invasion. The quantity of cell invasion and migration of miR-222 with/without overexpression group showed by Transwell assay was increased compared with negative control group, and the number of cell invasion and migration of miR-222 without overexpression group was decreased. These outcomes suggested that miR-200 might serve as a novel biomarker or therapeutic target for ovarian carcinoma.

$P T E N$, which is well recognized as a tumor suppressor gene in human cancer, has been found involved in the regulation of cancer cell biological behavior and playing an important part in the progression and development of various kinds of human cancer. PTEN has been reported as a target of miR-222 (21), Shen et al (24) reported that miR-222/PTEN/Akt/FOXO1 axis mediated ADR-resistance and prognosis of breast cancer patients. It was also suggested that miR-222 could decrease the sensitivity of breast cancer cells to adriamycin through PTEN/ Akt/p27 kip1 signaling pathway (25). In the present study, we determined PTEN as the target of miR-222 in ovarian carcinoma, a significantly increased expression of PTEN was observed after transfection by miR-222 mimic. However, miR-222 inhibitor decreased PTEN expression. Furthermore, to the best of our knowledge, for the first time, interference of PTEN was shown to promote migration and invasion. These studies together demonstrated that miR-222 might be a powerful anti-ovarian carcinoma candidate.

In conclusion, we revealed that miR-222 could promote ovarian carcinoma cell migration and invasion. PTEN was identified as a functional target of miR-222. The newly identified miR-222/PTEN may provide new insight into pathogenesis and represents a potential therapeutic target for ovarian carcinoma.

\section{Acknowledgements}

Not applicable.

\section{Funding}

No funding was received.

\section{Availability of data and materials}

The datasets used and/or analyzed during the present study are available from the corresponding author on reasonable request.

\section{Authors' contributions}

LG and WZ contributed to the conception of the study, YY contributed significantly to data analysis and manuscript preparation, XX performed the data analyses and wrote the manuscript, HL and GZ helped perform the analysis with constructive discussions. All authors read and approved the final manuscript.

\section{Ethics approval and consent to participate}

The study was approved by the Ethics Committee of People's Hospital of Rizhao (Rizhao, China). All patients signed the informed consent.

\section{Consent for publication}

Not applicable.

\section{Competing interests}

The authors declare that they have no competing interests.

\section{References}

1. Nam EJ, Yoon H, Kim SW, Kim H, Kim YT, Kim JH, Kim JW and Kim S: MicroRNA expression profiles in serous ovarian carcinoma. Clin Cancer Res 14: 2690-2695, 2008.

2. Cittelly DM, Dimitrova I, Howe EN, Cochrane DR, Jean A, Spoelstra NS, Post MD, Lu X, Broaddus RR, Spillman MA, et al: Restoration of miR-200c to ovarian cancer reduces tumor burden and increases sensitivity to paclitaxel. Mol Cancer Ther 11: 2556-2565, 2012.

3. Pal MK, Jaiswar SP, Dwivedi VN, Tripathi AK, Dwivedi A and Sankhwar P: MicroRNA: A new and promising potential biomarker for diagnosis and prognosis of ovarian cancer. Cancer Biol Med 12: 328-341, 2015.

4. Lupia M and Cavallaro U: Ovarian cancer stem cells: Still an elusive entity? Mol Cancer 16: 64, 2017. 
5. Koutsaki M, Libra M, Spandidos DA and Zaravinos A: The miR-200 family in ovarian cancer. Oncotarget 8: 66629-66640, 2017.

6. Wang Y, Xu C, Wang Y and Zhang X: MicroRNA-365 inhibits ovarian cancer progression by targeting Wnt5a. Am J Cancer Res 7: 1096-1106, 2017.

7. Siegel RL, Miller KD and Jemal A: Cancer statistics, 2016. CA Cancer J Clin 66: 7-30, 2016.

8. Calin GA and Croce CM: MicroRNA signatures in human cancers. Nat Rev Cancer 6: 857-866, 2006.

9. Bartel DP: MicroRNAs: Target recognition and regulatory functions. Cell 136: 215-233, 2009.

10. Chan E, Prado DE and Weidhaas JB: Cancer microRNAs: From subtype profiling to predictors of response to therapy. Trends Mol Med 17: 235-243, 2011.

11. Farazi TA, Hoell JI, Morozov P and Tuschl T: MicroRNAs in human cancer. Adv Exp Med Biol 774: 1-20, 2013

12. Hata A and Lieberman J: Dysregulation of microRNA biogenesis and gene silencing in cancer. Sci Signal 8: re3, 2015.

13. Saumet A and Lecellier CH: MicroRNAs and personalized medicine: Evaluating their potential as cancer biomarkers. Adv Exp Med Biol 888: 5-15, 2015.

14. Srivastava SK, Arora S, Averett C, Singh S and Singh AP: Modulation of microRNAs by phytochemicals in cancer: Underlying mechanisms and translational significance. BioMed Res Int 2015: 848710, 2015.

15. Virant-Klun I, Ståhlberg A, Kubista $M$ and Skutella T: MicroRNAs: From female fertility, germ cells, and stem cells to cancer in humans. Stem Cells Int 2016: 3984937, 2016.

16. Kinose Y, Sawada K, Nakamura K and Kimura T: The role of microRNAs in ovarian cancer. BioMed Res Int 2014: 249393 2014.

17. Wang Y, Kim S and Kim IM: Regulation of metastasis by microRNAs in ovarian cancer. Front Oncol 4: 143, 2014.

18. Garofalo M, Quintavalle C, Romano G, Croce CM and Condorelli G: miR221/222 in cancer: Their role in tumor progression and response to therapy. Curr Mol Med 12: 27-33, 2012.
19. Wei Y, Yang J, Yi L, Wang Y, Dong Z, Liu Z, Ou-Yang S, Wu H, Zhong Z, Yin Z, et al: MiR-223-3p targeting SEPT6 promotes the biological behavior of prostate cancer. Sci Rep 4: 7546, 2014.

20. Fu X, Li Y, Alvero A, Li J, Wu Q, Xiao Q, Peng Y, Hu Y, Li X, Yan W, et al: MicroRNA-222-3p/GNAI2/AKT axis inhibits epithelial ovarian cancer cell growth and associates with good overall survival. Oncotarget 7: 80633-80654, 2016.

21. Li B, Lu Y, Wang H, Han X, Mao J, Li J, Yu L, Wang B, Fan S, $\mathrm{Yu} \mathrm{X}$, et al: miR-221/222 enhance the tumorigenicity of human breast cancer stem cells via modulation of PTEN/Akt pathway. Biomed Pharmacother 79: 93-101, 2016.

22. Fang G, Liu J, Wang Q, Huang X, Yang R, Pang Y and Yang M: MicroRNA-223-3p regulates ovarian cancer cell proliferation and invasion by targeting SOX11 expression. Int J Mol Sci 18 pii: E1208, 2017.

23. Xu J, Jiang N, Shi H, Zhao S, Yao S and Shen H: miR-28-5p promotes the development and progression of ovarian cancer through inhibition of N4BP1. Int J Oncol 50: 2236, 2017.

24. Shen H, Wang D, Li L, Yang S, Chen X, Zhou S, Zhong S, Zhao J and Tang J: MiR-222 promotes drug-resistance of breast cancer cells to adriamycin via modulation of PTEN/Akt/FOXO1 pathway. Gene 596: 110-118, 2017

25. Wang DD, Yang SJ, Chen X, Shen HY, Luo LJ, Zhang XH, Zhong SL, Zhao JH and Tang JH: miR-222 induces adriamycin resistance in breast cancer through PTEN/Akt/p27kip1 pathway. Tumour Biol 37: 15315-15324, 2016.

This work is licensed under a Creative Commons Attribution-NonCommercial-NoDerivatives 4.0 International (CC BY-NC-ND 4.0) License. 